\title{
AN OVERVIEW ON HYPERSONIC FLOW RESEARCH WITH INFRARED THERMOGRAPHY
}

\author{
by Gennaro Cardone*
}

*Università di Napoli Federico II, DETEC, p.le Tecchio 80, 80125 Naples (Italy)

\begin{abstract}
The technological development achieved in instruments and methodology concerning both flights and ground hypersonic experiment (employed in space plane planning) goes towards an updating and a standardization of the heat flux technical measurements. In fact, the possibility to simulate high enthalpy flow relative to reentry condition by hypersonic arc-jet facility needs devoted methods to measure heat fluxes. Aim of this work is to demonstrate that InfraRed (IR) thermographic measurements with new heat flux sensor (IR-HFS) can be used as powerful tool in hypersonic high enthalpy flow research.
\end{abstract}

\section{Introduction}

In the modern hypersonic aero-thermodynamic design all the components of the spatial vehicle (wings, propulsion, fuselage, Thermal Protection System) are strongly coupled with each other and the acquisition of the technologies necessary for the development of a space plane requires both flight and ground experiments. In particular, the design and the optimization of the Thermal Protection System, TPS are critical aspects either to ensure the structural integrity and habitability of the hypersonic vehicle or to improve its aerodynamic performances. This goal needs aero-thermodynamic heating measurement and catalytic evaluation of the TPS materials. The possibility to simulate the hypersonic heat flux loads in order to test material and to solve any criticality regarding shapes, local heating and aerothermodynamic details, is one of the main goal of the actual re-entry simulator facilities.

Usually, measuring convective heat fluxes requires both a sensor (with its corresponding thermal model) and some temperature measurements. In the ordinary techniques, where temperature is measured by thermocouples, resistance temperature detectors or pyrometers, each transducer yields the heat flux at a single point, or in the space-averaged region of the same one; hence, in terms of spatial resolution, the sensor itself can be considered as zero-dimensional. This constraint makes experimental measurements particularly troublesome whenever temperature, and/or heat flux, fields exhibit high spatial gradients. The Infrared Scanning Radiometer (IRSR) constitutes a true two-dimensional temperature transducer since it allows the performance of accurate measurement of surface temperature maps even in the presence of relatively high spatial temperature gradients. Correspondingly, the heat flux sensor may become two-dimensional. In particular, infrared thermography can be fruitfully employed to measure convective heat fluxes, in both steady and transient techniques [1]. The thermal map obtained by means of currently available computerized thermographic systems is formed through a large amount of pixels (20k to $300 \mathrm{k}$ and more) so that IRSR can be practically regarded as a two-dimensional array of thin films. However, unlike standard thin films, which have a response time of the order of microseconds, the typical response time of IRSR is of the order of $10^{-1} \div 10^{-2} \mathrm{~s}$. 
The applicability of IR thermography to a hypersonic arc jet facility needs also the analysis and the evaluation of the radiation emitted and absorbed by the plasma flow impacting the test sample which is the medium between the IR Thermograph system (detector and window) and the test sample surface. Following the results of this analysis a proper detector wavelength window and optics/filter have been determined in order to minimize the noise and any undesired source of disturbance [2].

Then, the classical thin film technique is examined and its inadequacy in high enthalpy hypersonic heat flux measurement is shown. A new physical-mathematical model of heat flux sensor based on experimental data measured by means of IRSR and on numerical resolution of Fourier's equation in the solid model, the IR-HFS [3] is proposed.

After the numerical validation of proposed IR-HFS, it is applied in two arc jet plasma wind tunnels: SCIROCCO at Centro Italiano Ricerche Aerospaziali CIRA and HEAT at CENTROSPAZIO. In the first facility, that is essentially devoted to study TPS materials of re-entry space vehicles, the NASDA HYFLEX tests are analysed. In the second one, that is devoted to aerodynamic study of re-entry space vehicles, a few tests that investigate a shock-wave/boundary-layer interaction experiment are shown.

\section{Analysis of the emission-transmittance of hypersonic plasma flow}

As well know, the IR thermography is based on the collection of the radiation emitted by a solid body of know emissivity. In order to evaluate any possible source of undesired radiation or environmental constrain that may affect the precision, the accuracy, and, in general, the applicability of IR thermography to a Plasma Wind Tunnel, PWT. The attention was focused only to the analysis of the medium existing between the IR scanner / window and the test sample surface, i.e. the plasma flow. More precisely was neglected, as source of possible noise, any environmental radiation because the IR scanner/window system is applied on the test chamber wall in a protected box.

In order to obtain an evaluation and a quantitative estimation of the emission of plasma gas mixture, one need a physical model that describe and compute, starting from a full set of molecular and atomic transition lines, the complete radiation profile. The performing of this purpose, that represents the real case of non equilibrium radiation from hypersonic plasma gaseous flow, is an hard work and it is out from our aims in this phase of IR thermography feasibility study. In the literature there exist some attempts to give a theoretical model to the problem of the determination and calculation of radiation emitted by a non equilibrium plasma hypersonic flow such as the NEQAIR [4] code by Mr. C. Park and a similar code supported by ESA, the PARADE program. However the research in this field is still far from a complete understanding of all chemical and physical mechanisms involved in a plasma gas far from the thermo-fluid dynamical equilibrium. In particular the above mentioned models describe not the entire set but only the most important plasma components and the relative chemical reactions and energy level transitions.

To take into account more chemical species, the problem was approached in the classical LTE (Local Thermodynamic Equilibrium) hypothesis that is probably verified in the spatial region after the shock layer in front of the test sample surface, and by considering one chemical component at time.

In order to evaluate the presence and the relative concentration of the chemical species present in the plasma flow in the line of sight between the thermograph and the test sample, a detailed estimation of all possible components of 


\section{http://dx.doi.org/10.21611/qirt.2006.b}

the plasma gas has carried out by using two different computation codes: ARCSIZ by Aerotherm Co. and H2NS by CIRA. The first one is the code used in the design phase by the SCIROCCO Process Integrator and it is characterized by all possible combinations and chemical reaction of the four basic atomic components Carbon, Nitrogen, Oxygen and Argon. This code have also the possibility to take into account a simple model to introduce the non-equilibrium aspect by considering the chemistry frozen from a certain value of the Mach number ranging between 1.3 and 3.6, depending from the boundary condition. As alternative, many simulations by using the most sophisticated H2NS code [5], characterized by a more complex modelling of the non-equilibrium but mainly describing only the most important components of the air plasma gas (i.e. $\mathrm{O}, \mathrm{N}, \mathrm{NO}, \mathrm{O}_{2}$ and $\mathrm{N}_{2}$ ) have also performed (for example, if it is used the model named Park-83 [4], the considered chemical reaction are 18).

In table 1 a typical results of one ARCSIZ simulation $\left(H_{0}=20 \mathrm{MJ} / \mathrm{Kg}, \mathrm{P}_{0}=1\right.$ bar, Mach=7.5) is reported with all chemical components and their relative concentration in terms of molar fraction.

Table 1. Computed concentration (molar fraction) of a typical running condition in Plasma Wind Tunnel

\begin{tabular}{|c|c|c|}
\hline $\begin{array}{c}\text { Chemical } \\
\text { Specie }\end{array}$ & $\begin{array}{c}\text { Molar } \\
\text { fraction } \\
\text { before the } \\
\text { shock }\end{array}$ & $\begin{array}{c}\text { Molar } \\
\text { fraction } \\
\text { after the } \\
\text { shock }\end{array}$ \\
\hline $\mathrm{C}$ & $9,83 \mathrm{E}-08$ & $9,04 \mathrm{E}+00$ \\
\hline $\mathrm{AR}$ & $1,56 \mathrm{E}+03$ & $1,22 \mathrm{E}+03$ \\
\hline $\mathrm{CN}$ & $2,17 \mathrm{E}-07$ & $2,46 \mathrm{E}-01$ \\
\hline $\mathrm{CNN}$ & $3,02 \mathrm{E}-15$ & $2,81 \mathrm{E}-07$ \\
\hline $\mathrm{CO}_{2}$ & $5,18 \mathrm{E}-01$ & $2,09 \mathrm{E}-04$ \\
\hline $\mathrm{C}_{2} \mathrm{~N}_{2}$ & $2,69 \mathrm{E}-20$ & $1,88 \mathrm{E}-11$ \\
\hline $\mathrm{C}_{3} \mathrm{O}_{2}$ & $1,51 \mathrm{E}-24$ & $7,46 \mathrm{E}-19$ \\
\hline $\mathrm{C}_{5}$ & $1,37 \mathrm{E}-51$ & $1,24 \mathrm{E}-28$ \\
\hline $\mathrm{C}_{8}$ & $2,82 \mathrm{E}-88$ & $9,89 \mathrm{E}-52$ \\
\hline $\mathrm{NO}_{2}$ & $1,58 \mathrm{E}-03$ & $7,26 \mathrm{E}-06$ \\
\hline $\mathrm{N}_{2} \mathrm{O}_{3}$ & $2,19 \mathrm{E}-15$ & $2,20 \mathrm{E}-17$ \\
\hline $\mathrm{N}_{3}$ & $3,07 \mathrm{E}-08$ & $3,06 \mathrm{E}-04$ \\
\hline $\mathrm{N}_{2}$ & $6,45 \mathrm{E}+04$ & $2,97 \mathrm{E}+04$ \\
\hline $\mathrm{N}$ & $2,05 \mathrm{E}+01$ & $4,19 \mathrm{E}+04$ \\
\hline $\mathrm{NO}_{2}$ & $7,04 \mathrm{E}+02$ & $7,22 \mathrm{E}+01$ \\
\hline $\mathrm{NCN}_{2}$ & $2,51 \mathrm{E}-13$ & $2,83 \mathrm{E}-06$ \\
\hline $\mathrm{C}_{2}$ & $1,24 \mathrm{E}-18$ & $1,60 \mathrm{E}-06$ \\
\hline & & \\
\hline
\end{tabular}

\begin{tabular}{|c|c|c|}
\hline $\begin{array}{c}\text { Chemical } \\
\text { Specie }\end{array}$ & $\begin{array}{c}\text { Molar } \\
\text { fraction } \\
\text { before the } \\
\text { shock }\end{array}$ & $\begin{array}{c}\text { Molar } \\
\text { fraction } \\
\text { after the } \\
\text { shock }\end{array}$ \\
\hline $\mathrm{C}_{2} \mathrm{O}$ & $5,79 \mathrm{E}-15$ & $2,16 \mathrm{E}-08$ \\
\hline $\mathrm{C}_{4}$ & $2,03 \mathrm{E}-41$ & $8,85 \mathrm{E}-22$ \\
\hline $\mathrm{C}_{6}$ & $1,30 \mathrm{E}-64$ & $1,92 \mathrm{E}-36$ \\
\hline $\mathrm{C}_{9}$ & $1,67 \mathrm{E}-98$ & $6,77 \mathrm{E}-59$ \\
\hline $\mathrm{NO}_{3}$ & $2,12 \mathrm{E}-11$ & $4,23 \mathrm{E}-14$ \\
\hline $\mathrm{N}_{2} \mathrm{O}_{4}$ & $4,61 \mathrm{E}-22$ & $2,60 \mathrm{E}-25$ \\
\hline $\mathrm{O}_{3}$ & $1,01 \mathrm{E}-06$ & $1,74 \mathrm{E}-09$ \\
\hline $\mathrm{O}_{2}$ & $8,63 \mathrm{E}+02$ & $5,89 \mathrm{E}-01$ \\
\hline $\mathrm{O}$ & $3,23 \mathrm{E}+04$ & $2,71 \mathrm{E}+04$ \\
\hline $\mathrm{NCO}$ & $9,84 \mathrm{E}-09$ & $7,09 \mathrm{E}-06$ \\
\hline $\mathrm{CO}$ & $5,43 \mathrm{E}+01$ & $3,35 \mathrm{E}+01$ \\
\hline $\mathrm{C}_{2} \mathrm{~N}$ & $1,58 \mathrm{E}-18$ & $3,43 \mathrm{E}-08$ \\
\hline $\mathrm{C}_{3}$ & $4,69 \mathrm{E}-28$ & $3,15 \mathrm{E}-13$ \\
\hline $\mathrm{C}_{4} \mathrm{~N}_{2}$ & $3,58 \mathrm{E}-43$ & $7,09 \mathrm{E}-26$ \\
\hline $\mathrm{C}_{7}$ & $3,18 \mathrm{E}-75$ & $8,25 \mathrm{E}-44$ \\
\hline $\mathrm{C}_{10}$ & $4,29 \mathrm{E}-112$ & $4,22 \mathrm{E}-67$ \\
\hline $\mathrm{N}_{2} \mathrm{O}$ & $6,09 \mathrm{E}-04$ & $2,67 \mathrm{E}-04$ \\
\hline & & \\
\hline
\end{tabular}

By using both codes, the estimation of all chemical species and relative concentration for the entire set of flow conditions defining the typical operative map of a PWT facility (in particular, it is used the operative map of SCIROCCO PWT) was carried out. Then, it is assumed worthy of consideration any chemical species which results to be present with a significant value of concentration in terms of molar fractions. In table 2, the chemical species, present in not negligible amount at the least in one functioning PWT flow condition, are reported. 
The analysis about the behaviour of such a plasma gas regarding emission and absorption of the radiation in the infrared region is performed by considering a gas mixture like the sum of the chemical species reported in Table 2 representing it the worst case. The objective is to estimate the emission and absorption in IR spectrum in order to select a wavelength window where is possible to perform correctly the IR thermography measurements. The analysis is focused on the shortwave window $(2-6 \mu \mathrm{m})$ and long-wave window $(8-14 \mu \mathrm{m})$, where commercial IR systems are available.

Table 2. Computed highest concentration (molar fraction) by considering the whole operative running conditions in Scirocco Plasma Wind Tunnel.

\begin{tabular}{|c|c|}
\hline $\begin{array}{c}\text { Chemical } \\
\text { Specie }\end{array}$ & $\begin{array}{c}\text { Highest } \\
\text { concentration }\end{array}$ \\
\hline $\mathrm{N}_{2}$ & $7.8 \mathrm{E}-1$ \\
\hline $\mathrm{O}_{2}$ & $2.2 \mathrm{E}-1$ \\
\hline $\mathrm{N}$ & $4.5 \mathrm{E}-1$ \\
\hline $\mathrm{O}$ & $3.5 \mathrm{E}-1$ \\
\hline $\mathrm{Ar}$ & $2,00 \mathrm{E}-02$ \\
\hline $\mathrm{NO}$ & $2,00 \mathrm{E}-02$ \\
\hline $\mathrm{CO}{ }_{2}$ & $1,00 \mathrm{E}-03$ \\
\hline $\mathrm{CO}$ & $1,00 \mathrm{E}-03$ \\
\hline $\mathrm{C}$ & $1,00 \mathrm{E}-03$ \\
\hline $\mathrm{NO}_{2}$ & $1,00 \mathrm{E}-05$ \\
\hline $\mathrm{CN}$ & $1,00 \mathrm{E}-05$ \\
\hline $\mathrm{N}_{2} \mathrm{O}$ & $1,00 \mathrm{E}-06$ \\
\hline $\mathrm{O}_{3}$ & $1,00 \mathrm{E}-07$ \\
\hline
\end{tabular}

To perform the estimation of the spectral line intensity $S$ of the chemical species reported in table 2, the HITRAN [6] (acronym for High-Resolution Transmission Molecular Absorption) database is used. Then, from these line intensity distributions and taking into account the thermodynamical conditions (temperature and partial pressure) and the maximum plasma thickness (about one meter in SCIROCCO), the transmittance by using the E-TRANS code by Ontar Co is computed.

In Fig. 1 the intensity of considered species are reported at $1000^{\circ} \mathrm{K}$ for the IR short-wave and IR long-wave windows, respectively.

In Fig. 2 the transmittance in the whole infrared region $1-12 \mu \mathrm{m}$ is shown for two different temperatures of $300^{\circ} \mathrm{K}$ and $1000^{\circ} \mathrm{K}$, respectively.

These results indicate that the long-wave window $(8-14 \mu \mathrm{m})$ is the best solution for the application of the IR thermography to measure temperatures over sample surfaces immersed in hypersonic plasma flow tests. 


\section{Heat flux measurements}

Usually, in cold hypersonic wind tunnel, heat fluxes are widely measured with thin film, a calorimetric technique that measure heat fluxes in an indirect way by mathematical model that estimates the heat transfer rates by transient temperature measurement. The mathematical model of the thin film sensor is a simplified heat conduction model with the follow assumption: thermal penetration depth is small and

$S$

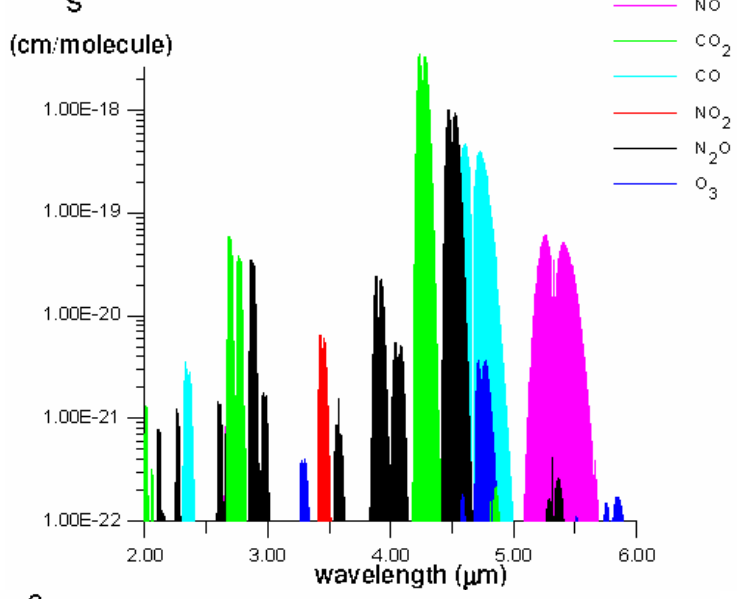

S

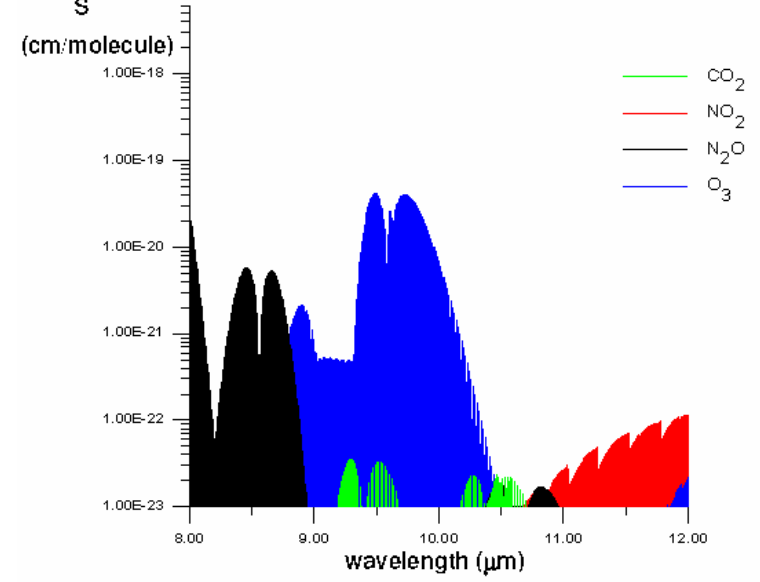

Fig. 1. Spectral line intensity $S$ in the short and long-wave window

therefore the wall may be assumed to behave as a semi infinite medium, material characteristic are independent of temperature, heat flux through normal medium surface is stronger then one in transversal directions therefore one dimensional hypothesis is satisfied. By assuming the thin-film sensor to be isothermal at initial time $t=0$, a suitable solution of heat conduction model to evaluate the heat flux from the measured surface temperature is [7]: 


$$
Q_{c}-Q_{r}=\sqrt{\frac{\rho c \lambda}{\pi}}\left[\frac{\phi(t)}{\sqrt{t}}+\frac{1}{2} \int_{0}^{t} \frac{\phi(t)-\phi(\xi)}{(t-\xi)^{3 / 2}} d \xi\right]
$$

where: $\phi$ is the temperature difference $T_{w}(t, 0)-T_{w i} . T_{w i}$ being the initial value of the wall temperature); $Q_{r}$ is the radiative heat flux to ambient; $Q_{c}$ is sum of convective and chemical heat flux; $\rho, c$ and $\lambda$, are the mass density, the specific heat and the thermal conductivity coefficient of the sensor material, respectively. The solution

a)

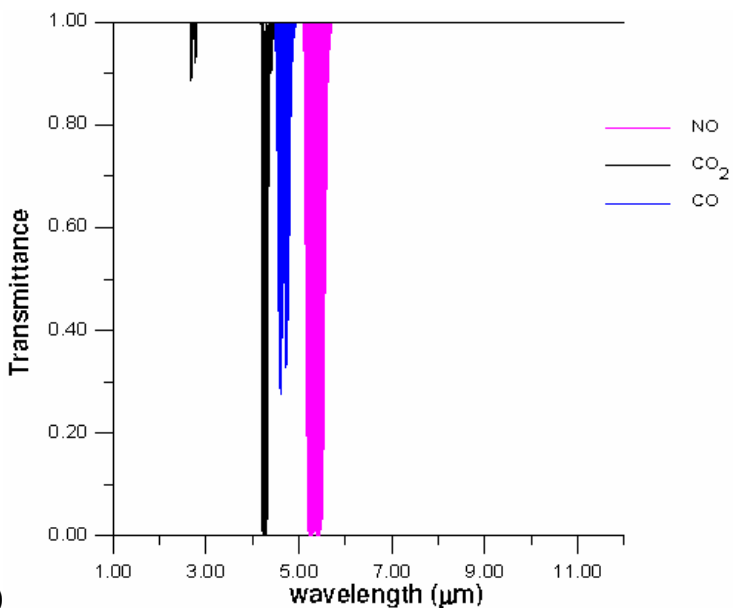

b)

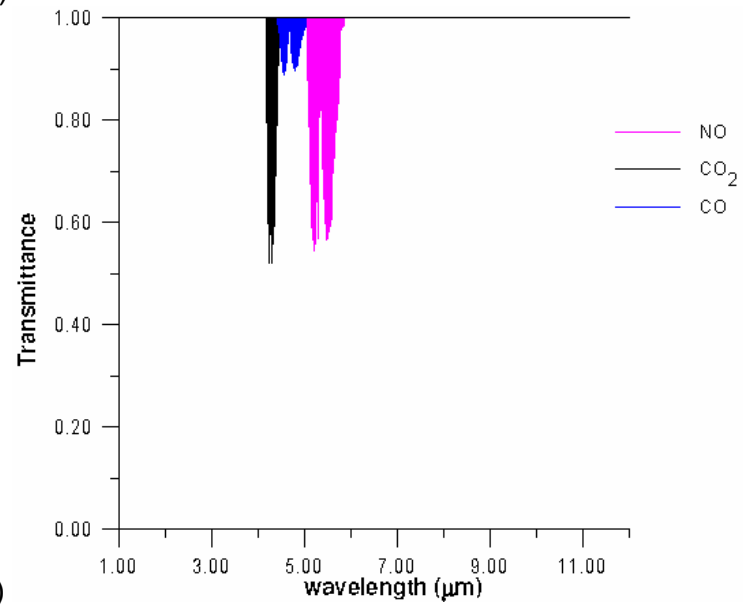

Fig.2. Transmittance profile at a) $300^{\circ} \mathrm{K}$ and b) $1000^{\circ} \mathrm{K}$

involves the numerical integration of the right terms and two numerical techniques are normally accepted for aerospace application: direct method [8], indirect method [9]. The first one computes the heat transfer rate directly from the unsteady wall temperature. The second one evaluates the wall heat flux over time and in subsequent moment determines its derivation respect to time. 
The use of classical thin film procedure in high enthalpy hypersonic wind tunnel, presents a lot of limits; the hypothesis of constant properties of is not satisfied; due to high noise of measurements the numerical integration of Eq. 1 is critical; the classical procedure do not take in account of injection time of model in the flow; the presence of radiative heat flux on model surface; IR surface temperature measurements are linked to surface properties and environments temperature. For this reason, in order to use IR measurements to evaluate heat fluxes in high enthalpy wind tunnel it is necessary to develop new heat flux sensor, IR-HFS to exceed the limit of classical thin film procedure.

\subsection{IR-HFS}

In this work it is proposed an alternative approach based on the assumption that the solution of direct problem yields a certain temperature time variation function of some free parameters. These parameters are found so that the computed temperatures best agree with the experimentally measured temperatures. The best fit may be determined by the ordinary least squares criterion [11]. In order to identify the free parameters of problem it is necessary to briefly describe the physical phenomena to be model: high enthalpy plasma flow around a body. The plasmamodel interaction involves all known forms of the heat fluxes: convection, surface catalysis, radiation and conduction inside body. The wall of test model is fully catalytic and it is assumed to know all its physical properties. The chemical reactions are catalysed at an infinite rate. The mass fractions at the wall are their local equilibrium values at local pressure and temperature. The thermal history of the body surface is indirectly acquired by infrared camera (Planck's law) and represents the experimental data.

The experimental data are function of the wall emissivity and radiative reference temperature $T_{r}$. In fact the temperatures measured by means of IR camera can be expressed as:

$$
T_{w}(t)=B / \ln \left(F+\frac{R}{\frac{I U(t)}{\varepsilon}-\frac{(1-\varepsilon)}{\varepsilon} I_{a m b}}\right)
$$

With $I U$ Isotherm Unit that represents the signal measured from the IR camera; $\varepsilon$ is the model emissivity; $R, B, F$ calibration constants; $I U_{a m b}=R /\left(\exp \left(B / T_{r}\right)-F\right)$ corresponding to $I U$ due to a black body at radiative reference temperature $T_{r}$. Typically the radiative reference temperature coincides with the ambient temperature but in our case it is function of innumerable factors like test chamber, fluid flow around the model, real thermo dynamical property of the plasma (non equilibrium) ecc.. It is assumed that the model emissivity $\varepsilon$ is a datum of the problem, instead the radiative reference temperature $T_{r}$ is an unknown parameter to found.

It is also necessary put attention to the phase of introduction of the model inside the test chamber. The necessary time $\Delta t_{i}$ to accomplish this phase determines a gap in experimental data and usually $\Delta t_{i}$ it is not a negligible part of acquisition time (a typical problem of all the large size wind tunnel facility like SCIROCCO). In this interval the temporal distribution of the $I U$ and as consequence the wall temperature, relative to each superficial point of the model, is unreal as direct consequence of the existent relative motion between the IR scanner and model and otherwise, during the initial phase the body crosses the plasma boundary layer where the thermo fluid dynamic parameters are different from free stream values. This unphysical behaviour 
is shown in Fig. 3. To exceed the gap in experiment data, the free parameters $T_{w i}$, $\Delta t_{i}$ are introduced.

The temperature inside the wall is solution of Fourier equation. In order to resolve the same equation the following hypothesis are made: heat flux through normal medium surface is stronger then one in transversal directions therefore the equation is one dimensional; thermal properties are function of temperature. If define this temperature $T^{\star}(t, x)$ the equation becomes:

$$
\frac{\partial}{\partial t} \int_{L} \rho c T^{*}(t, x) d x=\int_{L} \frac{\partial}{\partial x} \lambda \frac{\partial}{\partial x} T^{*}(t, x) d x
$$

The solution $T^{*}(t, x)$ is computed by means of Finite Volume method [11]. To resolve this equation it is necessary to fix the boundary condition.

The first is $T^{\star}(0, x)=T_{\text {wi }}$. If the measuring time $t_{M}$ verified that $t_{M}<\frac{L^{2}}{2 \alpha}$ where $\alpha$ is the TPS material thermal diffusivity coefficient and $L$ is the tickness of model, the boundary condition of adiabatic wall on back side of model can also used. The last one boundary condition is:

$$
Q_{k}=-\left.k \frac{\partial T^{*}}{\partial x}\right|_{x=0}
$$

with $Q_{k}$ the heating per unit area at wall model. By making a local energy balance it is possible to write $Q_{k}(t)=Q_{r}(t)+Q_{c}(t)$, where $Q_{r}$ is the radiative heat flux to ambient; $Q_{C}$ is sum of convective and chemical heat flux. Introducing some assumptions, the functional dependence of these fluxes from the free parameters of our problem, can be found.

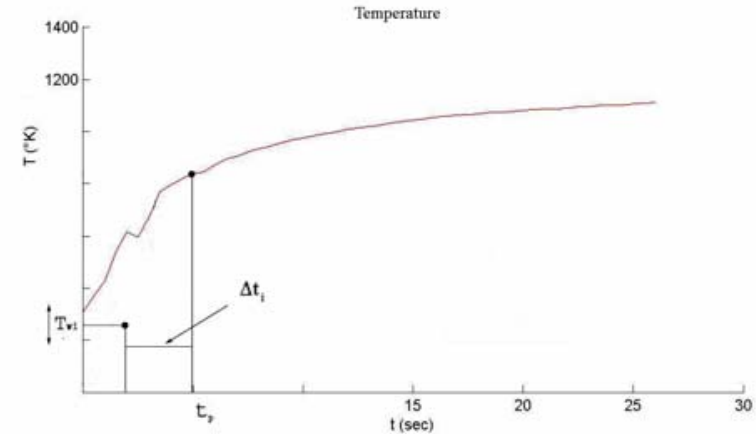

Fig 3. Example of experimental temperature time history

The radiative heat flux can be evaluated

$$
Q_{r}(t)=\sigma \varepsilon\left(T_{w}(t)^{4}-T_{r}^{4}\right)
$$




\section{http://dx.doi.org/10.21611/qirt.2006.b}

where: $\sigma$ is the Stefan-Boltzmann constant; $\varepsilon$ is the total emissivity coefficient; $T_{w}$ and $T_{r}$ are the temperature of the wall and the reference radiative temperature, respectively.

Following Newton's intuition it is introduced $h_{c}$, a local heat transfer coefficient:

$$
Q_{c}(t)=h_{c}\left(T_{w}(t)-T_{a w}\right)
$$

that include both convective and catalytic phenomena. The relationship (6) shows also the dependence of $Q_{c}$ from the adiabatic wall temperature $T_{a w}$. It is assumed that $h_{c}$ is function only of testing conditions.

In conclusion, the free parameter of our problem are: $\Delta t_{i}, h_{c}, T_{a w}, T_{r}, T_{w i}$. In the free parameters are found minimizing the functional:

$$
\mathfrak{J}\left(\Delta t_{i}, h_{c}, T_{a w}, T_{r}, T_{w i}\right)=\sum_{j=1}^{n}\left(T_{w j}-T_{j}^{*}\right)^{2}
$$

where $T_{w j}$ is the j-term of the $n$ experimentally measured surface temperature values and $T^{*}$ is the wall temperature predicted by Finite Volume method $[11,12]$. Both of these temperatures are evaluated at the same time and at the same location. The functional is minimized by the optimisation technique Levenberg-Marquardt. The adequacy of the proposed model to the physical phenomena is measured by evaluating the statistical correlation parameter between the predicted and measured temperatures.

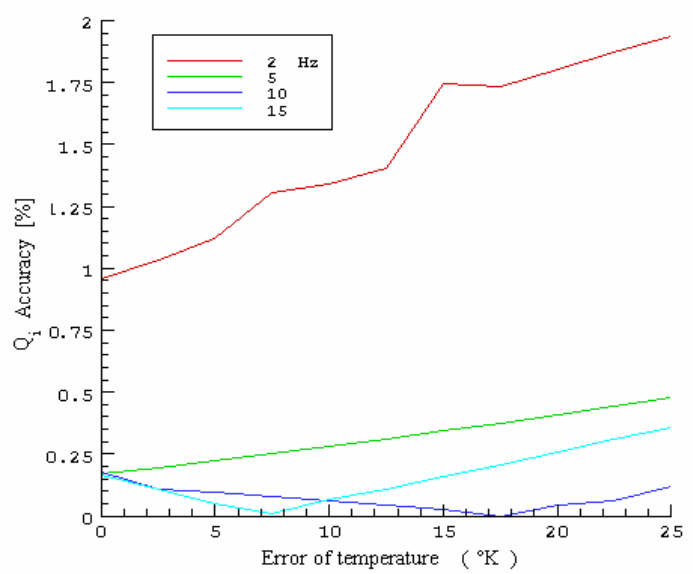

Fig 4. $Q_{i}$ Accuracy

\subsection{IR-HFS numerical validation}

In this phase the stability of the numerical algorithm and its accuracy is analysed. The first step is generating and perturbing with noise an ideal numerical solution of the same algorithm. Then the developed numerical procedure is applied to the perturbed solution and in the end comparing the determined values of the 
parameters with the ideal ones, the IR-HFS model can validated. To compute the ideal solution, the typical expected values of free parameters for hypersonic plasma flow and the typical experimental constants (model thermal property, IR calibration constants, etc) are used. Different values of acquisition frequencies $(2,5,10,15 \mathrm{~Hz})$ and a random noise temperature equal to $0 \div 25^{\circ} \mathrm{K}$ was also evaluated. The accuracy on the convective heat flux at $T_{w}=T_{w i}, Q_{i}$, is shown in Fig. 4. The Fig. 5 shows an example of perturbed ideal transient temperature and the corresponding estimated one.

\section{NASDA HYFLEX experiment}

The SCIROCCO arc-jet facility presents some peculiarities that make it unique in the world. In particular, the arc heater length and diameter are respectively of $5500 \mathrm{~mm}$ and $110 \mathrm{~mm}$ and its maximum power is $70 \mathrm{MW}$. The details of the facility design, the characteristics of the various components and the expected performances of the Scirocco PWT are described in $[13,14,15]$. Briefly, the SCIROCCO facility is a typical segmented-constrictor Arc-Jet Wind Tunnel (AWT). The gas used for the tests is dry compressed air with mass flow variable from 0.1 to $3.5 \mathrm{~kg} / \mathrm{sec}$. It accelerates through a convergent-divergent conical nozzle with interchangeable exit diameters up to $1950 \mathrm{~mm}$. The flow velocity at the nozzle exit can reach a value of $7000 \mathrm{~m} / \mathrm{s}$. A powerful Vacuum System working via the action of twelve steam ejectors is located downstream of the test chamber. During a test, after the stable flow condition is, in the test chamber, attained and confirmed by probe sensor, the testing model is put into the flow by the support system with characteristic time of $2 \div 5 \mathrm{sec}$.

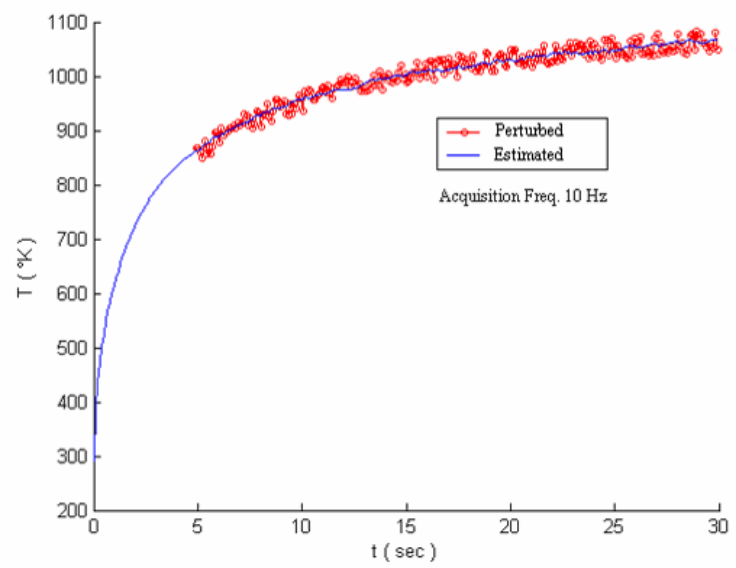

Fig. 5. Ideal and perturbed Temperature

The thermocamera used is the Agema Thermovision THV900LW. It is equipped with a Cd-Te-Hg detector scanning and its field of view holds in the IR waveband $8 \div 12 \mu \mathrm{m}$ with a frame of 136 not-interlaced lines in $1 / 15 \mathrm{~s}$. The thermal image is digitized with a resolution of $272 \times 136$ pixels that, in combination to a $10^{\circ} \times 20^{\circ}$ IR lens placed at the distance of about $4 \mathrm{~m}$ from the test sample, results in $a$ spatial resolution of about $1 \mathrm{~cm} /$ pixel. The thermocamera is mounted in a protected box on a robotized remotely controlled gear, in order to avoid any disturbance from ambient radiation. In front of the thermo camera a ZnSe IR windows with a special 


\section{http://dx.doi.org/10.21611/qirt.2006.b}

coating to reduce the band pass wavelength region to the proper values of about 8$10 \mu \mathrm{m}$ is installed.

The IR thermograph investigations presented in this section have been performed during the test campaign carried out in Scirocco on an instrumented test sample identical to the HYFLEX nose geometry [16]. The overall diameter is about $80 \mathrm{~cm}$ (radius $40 \mathrm{~cm}$ ), the heat shield layer is divided in eight tiles with a gap of about $1-2 \mathrm{~mm}$ and an hemispherical nose, shown in Fig.6. The inner shell structure is made of CFRP material as substrate while the TPS material is made by Zyalite ${ }^{\mathrm{tm}}$ moldable with a thickness of $30 \mathrm{~mm}$ and with surface treated with a black coating made with Tyranno $^{\odot}$ in order increase the emissivity of the surface and to reduce the possible bulk temperatures below the limit of the mouldable Zyalite material. The emissivity of the coating was measured by the National Aerospace Laboratory (Japan), Fig.7. The heat resistance capabilities and thermal characteristics of the heat shield materials were tested at plasma heating facility located at Japanese Ultra-high Temperature Research Institute (JUTEM) and an ANSYS parametric study on thermal response of Moldable also exists [17].

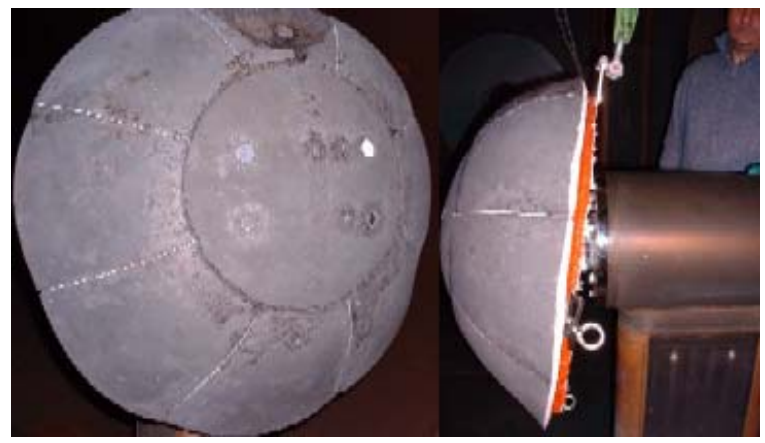

Fig. 6. NASDA Hyflex test sample. The instrumented sensors (temperature, heat flux and catalytic sensors) can be noticed on the stagnation hemispherical nose.

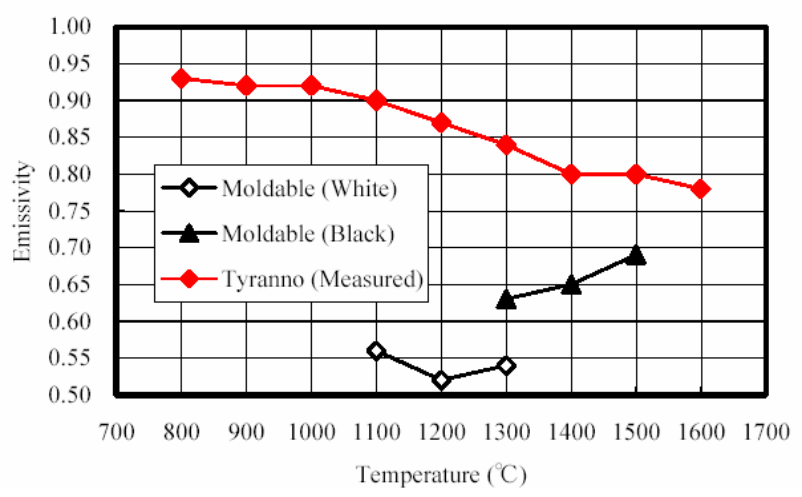

Fig. 7. Measured emissivity vs Temperature for the test sample surface (red squares).

The test campaign reproduced four flow conditions, two typical of the normal orbital reentry condition $\mathrm{H} 1, \mathrm{H} 2$ and other two based on the flight trajectory of HYFLEX vehicle F1, F2. In the Table 3 main flow parameters measured during the 
four test cases are reported. The test duration was fixed to around 30 seconds in order to preserve the heat shield material of the sample.

Table 3. Main flow parameters in HYFLEX tests.

\begin{tabular}{|l|l|l|l|l|}
\cline { 2 - 5 } \multicolumn{1}{c|}{} & $\mathrm{H} 1$ & $\mathrm{H} 2$ & $\mathrm{~F} 1$ & $\mathrm{~F} 2$ \\
\hline $\mathrm{H}_{0}[\mathrm{MJ} / \mathrm{Kg}]$ & 11.9 & 13.3 & 7.5 & 8.7 \\
\hline $\mathrm{P}_{0}[\mathrm{bar}]$ & 2.45 & 2.40 & 7.45 & 8.00 \\
\hline
\end{tabular}

A picture of the HYFLEX test sample under test condition $\mathrm{H} 1$ is shown in Fig.8, from side point of view. The thermal map recorded at the end of the exposure time to the plasma flow is reported in Fig.9. On the same one the presence of the two catalytic sensors, realized by different materials with respect the heat shield, are visible as the two circular and symmetrical cold spots.

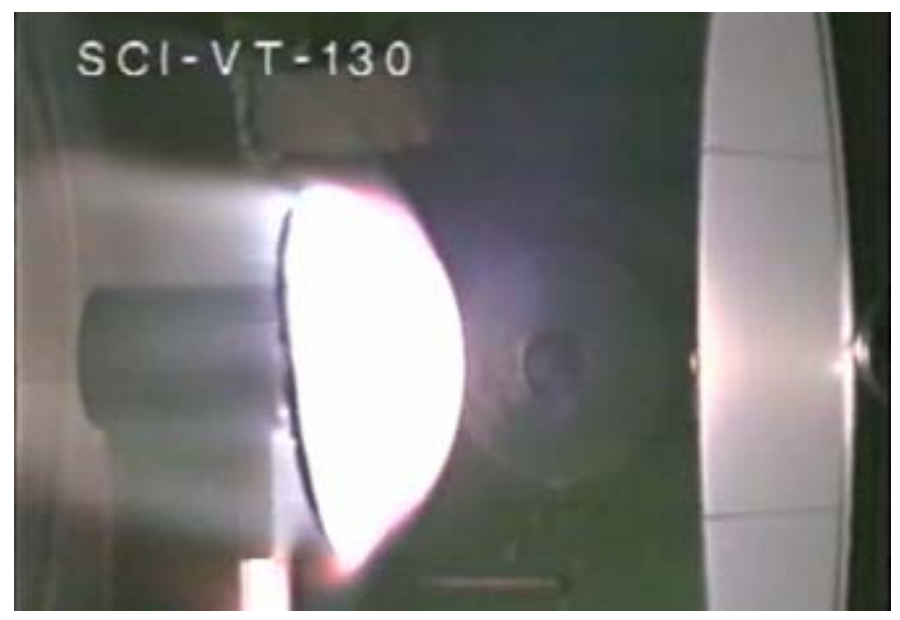

Fig. 8. NASDA Hyflex test sample under test in flow condition H1 (Table 1)

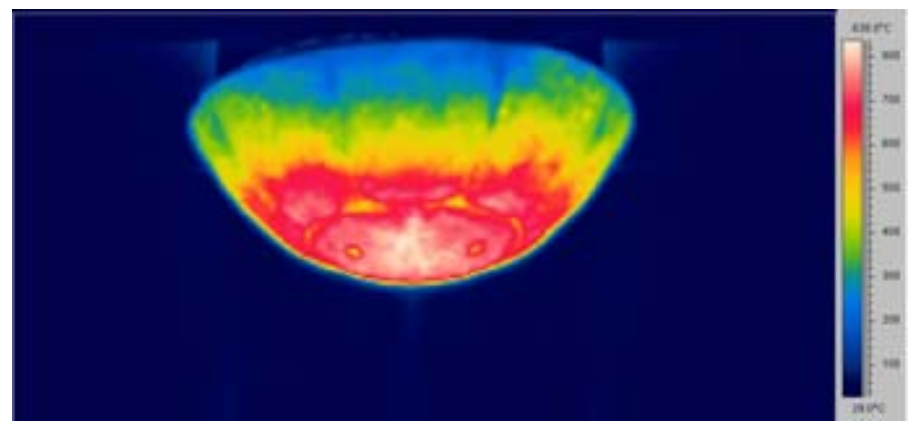

Fig. 9. IR Thermo camera images of the NASDA Hyflex test sample under test in flow condition F2.

The IR-HFS has been applied to the experimental data acquired during the HYFLEX tests carried out at CIRA. The Fig.10 shows the measured and predicted 


\section{http://dx.doi.org/10.21611/qirt.2006.b}

time history of temperature relative to the stagnation point in the case of $\mathrm{F} 2$ test. The high value of correlation factor indicates the adequacy of the phenomenological assumptions made in IR-HFS. In Table 4 are reported the predicted heat fluxes by means of IR-HFS in the stagnation point for the four HYFLEX test cases at two different wall temperature. In the same table it is also reported the heat fluxes measured by [17] with standard measurements and computed by CIRA with CFD [18].

\section{Shock-wave/boundary-layer interaction experiment}

The development of hypersonic vehicles has renewed the attention on the problem of viscous inviscid flow interactions and, in particular, on shockwave/boundary-layer interaction phenomena that are of great practical importance for air-breathing engine inlets, wing/body junctures and deflected control surfaces. Prediction of thermal and dynamic loads on surfaces exposed to hypersonic flows is an essential prerequisite for the effective design of aerodynamic control surfaces and of thermal protection system of modern space vehicles in their trans-atmospheric flight portion.

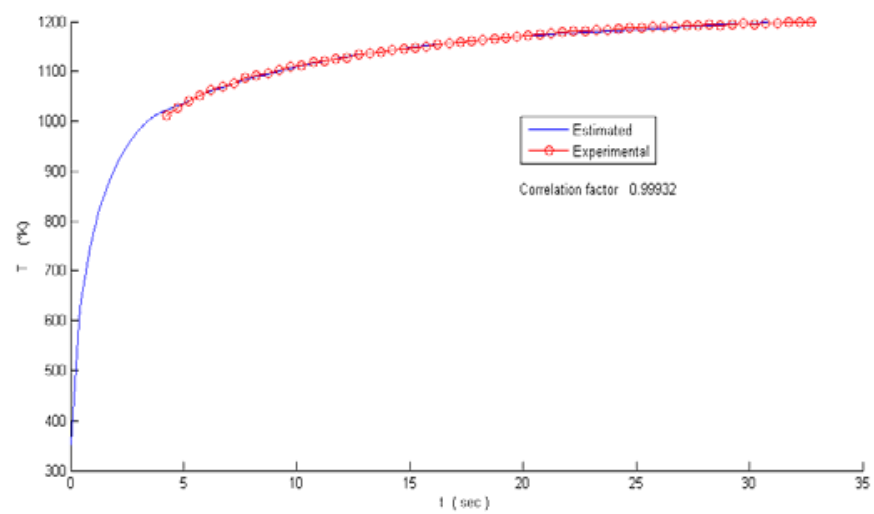

Fig. 10. Measured and predicted temperatures

Table 4. Heat Fluxes Comparison

\begin{tabular}{|l|c|c|c|c|c|}
\hline \multirow{2}{*}{$\begin{array}{c}\text { Test Condition } \\
H_{0}[\mathrm{MJ} / \mathrm{Kg}], P_{0}[\mathrm{Bar}], T w\end{array}\left[^{\circ} \mathrm{K}\right]$} & \multicolumn{3}{|c|}{$\begin{array}{c}\text { Stagnation Heat Fluxes } \\
{\left[\mathrm{KW} / \mathrm{m}^{2}\right]}\end{array}$} & \multicolumn{2}{c|}{$\begin{array}{c}\text { Error respect CIRA } \\
\%\end{array}$} \\
\cline { 2 - 7 } & IR HFS & CIRA & NASDA & IR HFS & NASDA \\
\hline$H_{0}=11.9 ; P_{0}=2.45 ; T_{w}=300$ & 149,2 & 141 & 191 & 5,82 & 35,94 \\
\hline$H_{0}=1.9 ; P_{0}=2.45 ; T_{w}=1108$ & 123,8 & 127,7 & --- & $-3,05$ & --- \\
\hline$H_{0}=13.3 ; P_{0}=2.40 ; T_{w}=300$ & 184,7 & 156,9 & 203 & 17,71 & 29,38 \\
\hline$H_{0}=13.3 ; P_{0}=2.40 ; T_{w}=1143$ & 153,4 & 144,4 & --- & 6,23 & --- \\
\hline$H_{0}=7.5 ; P_{0}=7.45 ; T_{w}=300$ & 144,9 & 147,7 & 123 & $-1,89$ & $-16,72$ \\
\hline$H_{0}=7.5 ; P_{0}=7.45 ; T_{W}=1103$ & 115,6 & 125,6 & --- & $-7,96$ & --- \\
\hline$H_{0}=8.7 ; P_{0}=8.0 ; T_{w}=300$ & 188,5 & 180,2 & 184 & 4,61 & 2,11 \\
\hline$H_{0}=8.7 ; P_{0}=8.0 ; T_{w}=1175$ & 154,7 & 155,2 & --- & $-0,32$ & --- \\
\hline
\end{tabular}


Measurements presented in this section refer to shock wave-boundary layer interaction in a two-dimensional hypersonic flow over a model consisting of a flat plate followed by a compression ramp (wedge) with its hinge line parallel to the model's leading edge.

The model surface is realized with two separate MACOR ${ }^{T M}$ plates screwed onto aluminium supports. The model spanwise dimension is $100 \mathrm{~mm}$. The hinge line is positioned at $50 \mathrm{~mm}$ from the leading edge and the ramp angle is $15^{\circ}$. MACOR ${ }^{\mathrm{TM}}$ was chosen as the model surface material for its low thermal conductivity, as required in connection with the use of thin film model.

Experimental tests have been carried out in Centrospazio High-Enthalpy Archeated Tunnel (HEAT) $[19,20]$ that is capable of producing Mach 6 flows with a specific total enthalpy up to $2.5 \mathrm{MJ} / \mathrm{kg}$ on an effective test section $60 \mathrm{~mm}$ in diameter, in the low to medium Reynolds number range $\left(10^{4}-10^{6}\right)$. The tunnel operates in a pulsed, quasi-steady mode, with running time ranging from 50 to 200ms. HEAT facility mainly consists of an arc gas heater and a contoured expansion nozzle, installed in a vacuum chamber volume of $4.1 \mathrm{~m}^{3}$; auxiliary systems are fitted to the arc heater to provide it with working fluid and energy. Four rotary pumps evacuate the chamber until an ultimate pressure of $10 \mathrm{~Pa}$ is reached before each run. This vacuum level allows an under-expanded hypersonic flow-field to be maintained at the nozzle exit for a running time longer than $200 \mathrm{~ms}$. IR camera used during the test was FLIR SC 3000 and acquisition frame frequencies was $60 \mathrm{~Hz}$ for flow visualization and $300 \mathrm{~Hz}$ for heat flux measurements.

A thermal map recorded about $80 \mathrm{~ms}$ after the starting of wind tunnel is reported in Fig. 11. The temperature distribution is almost bidimensional only near the model leading edge (the flow comes from left to right). Moving downwind, the continuous decrease of wall temperature, indicates the development of the boundary layer. Near the hinge line is clearly visible a region where the temperature attains a minimum that is due to the presence of a separation region in the flow. Moving along the symmetry axis after the hinge line the temperature reaches a maximum that is to be correlated to the flow reattachment on the ramp.
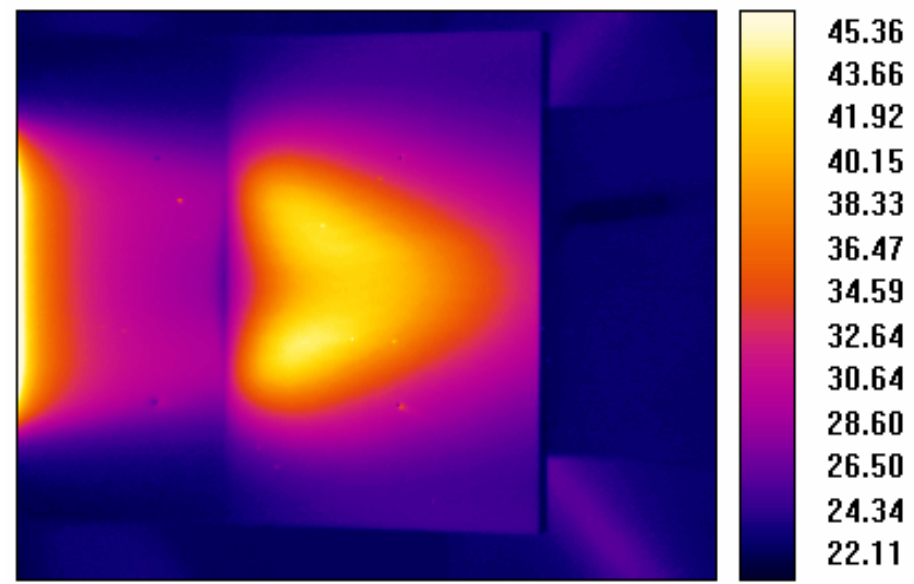

Fig. 11. Temperature map (in $\left.{ }^{\circ} \mathrm{C}\right)$ recorded on model surface after $80 \mathrm{~ms}$ from tunnel starting. Total enthalpy:2.3 MJ/Kg; Stagnation pressure:4.6 bar 


\section{http://dx.doi.org/10.21611/qirt.2006.b}

If one considers that, in a first approximation, the potential core may be assimilated to a cone emerging from the nozzle exit (the cone height being determined by the expansion fan angle at the nozzle exit, ideally starting at arcsin $(1 / M), M$ Mach number), the intersection of this cone with model surface is clearly visible on the thermal map. The measured temperature time histories are used to compute heat flux with thin film model described in section 3 . In this case it was not possible to use the alternative approach proposed in because during the first $30 \mathrm{~ms}$ of test run the total enthalpy (and therefore the reference temperature) is not constant. For two typical runs, in Fig. 12 the heat flux along the symmetry axis is presented in non-dimensional form by means of the Stanton number based on the adiabatic wall temperature computed by means of the recovery factor for laminar boundary layer flow [10].

Experimental data are also compared with the classical flat plate boundary layer analytical solution [9]. The results show a good agreement with theoretical solution on the flat plate. Near the hinge line (leading edge distance, $X=50 \mathrm{~mm}$ ) the presence of a separation region is clearly identified from the minimum of the Stanton number distribution. The entity of the heat flux at reattachment is in good agreement with data present in literature.

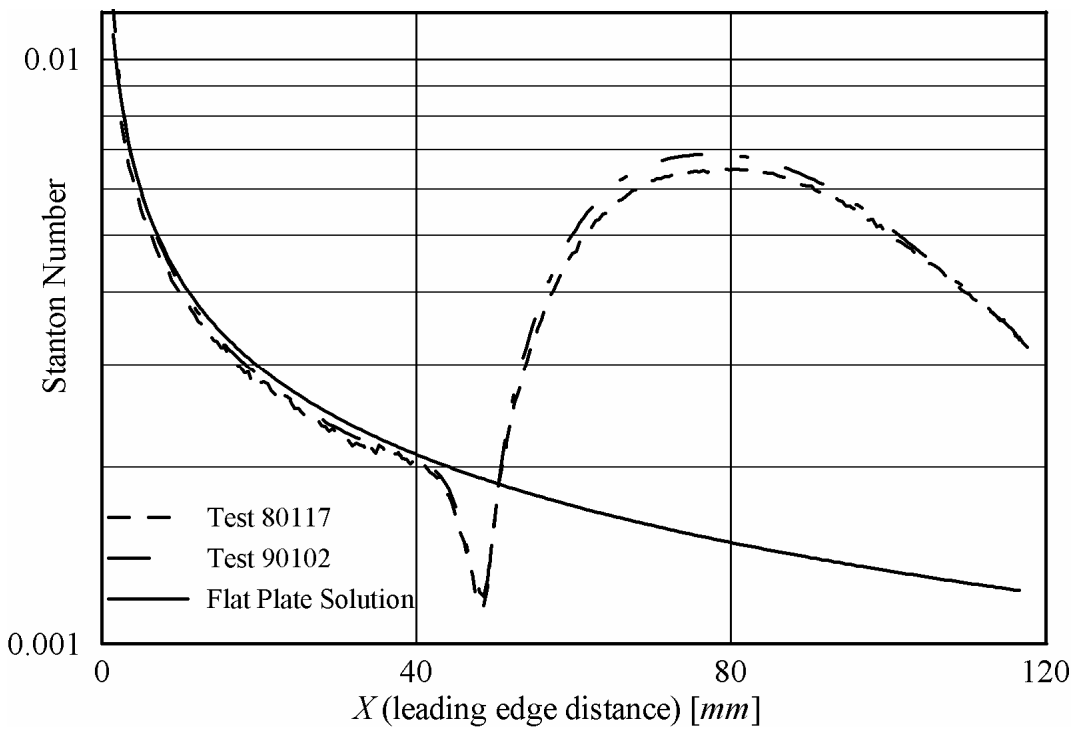

Fig. 12. Stanton number profile on symmetry axis. Total enthalpy:1.8MJ/Kg; Stagnation pressure: 6 bar

\section{Conclusions}

The technical and experimental aspects for the application of the IR thermography to measure temperature and heat flux over the surface test sample immersed in a hypersonic plasma flow are discussed and analyzed. The experimental results of the temperature measurements by IR thermography are reported in the case of two different application in plasma wind tunnels. The results demonstrate that the IR thermography represent a real two dimensional, spatially resolved, and non-intrusive powerful diagnostic technique for temperature 
measurements. The limits of classical thin film procedure in high enthalpy hypersonic wind tunnel have been shown and a new heat flux sensor based on experimental data measured by means of IRSR and on numerical resolution of Fourier's equation in the solid model, the IR-HFS is proposed. This sensor is based on the assumption that the solution of direct problem yields a certain temperature time variation function of some free parameters, then such parameters are found so that the computed temperatures best agree with the experimentally measured temperatures. A numerical validation of IR-HFS is performed in order to evaluate the accuracy and the stability of proposed procedure. The heat transfer measurements performed in two different experiments are in good agreements with numerical and analytical predictions.

\section{REFERENCES}

[1] T. Astarita, G. Cardone, G. M. Carlomagno, Infrared thermography: An optical method in heat transfer and fluid flow visualisation, Optics and Lasers in Engineering, Volume 44, (2006) 261-281.

[2] A. Del Vecchio, G. Cardone, IR thermographic measurements of temperatures in hypersonic large-scale plasma flow, 12th AIAA International Space Planes and Hypersonic Systems and Technologies, AIAA paper 20036926 (2003).

[3] G. Cardone, G. Tortora. and A. Del Vecchio. IR Thermographic measurements of termperatures and heat fluxes in hypersonic plasma flow. 5th European Symposium on Aerothermodynamics for Space Vehicles (2004).

[4] C. Park, Nonequilibrium Air Radiation (NEQAIR) Program: User's Manual, NASA TM-86707 (1985).

[5] F. De Filippis, A. Schettino, M. Serpico, S. Borrelli, Complete Analytical Model to Describe the Test-Leg of Scirocco PWT, $20^{\text {th }}$ Congress of the International Council of the Aeronautical Science, Sorrento, Italy (1996).

[6] L.S. Rothman, et al., The HITRAN molecular spectroscopic database: edition of 2000 including updates through 2001, Journal of Quantitative Spectroscopy \& Radiative Transfer, N. 82, (2003) 5-44.

[7] H.S. Carslaw and J.C. Jaeger. Conduction of heat in solids. Oxford University Press (1959).

[8] W.J. Cook, and E.J. Felderman, Reduction of Data from Thin-Film HeatTransfer Gages: A Concise Numerical Technique, AIAA. J., vol. 4, (1966) 561-562.

[9] G. Simeonides, Hypersonic shock wave boundary layer interactions over compression corners, PhD Thesis, University of Bristol - Faculty of Engireering Dip. of Aerospace Engineering, (1992).

[10] L. De Luca, G. Cardone, D. Aymer de la Chevalerie and A. Fonteneau, Experimental Analysis of Viscous Interaction in Hypersonic Wedge Flow, AIAA J., vol. 33 No. 12 (1995) 2293-2298.

[11] C. Hirsch, Numerical Computation of Internal and External Flows, Volume 1 Fundamental of Numerical Discretizazion, John Wiley \& Sons (1994).

[12] H.K. Versteeg, W. Malalasekera, An introduction to computational fluid dynamics, The Finite Volume Method, John Wiley \& Sons (1995).

[13] F. De Filippis, S. Caristia, and A. Del Vecchio, The Scirocco facility: Qualification phase, $2^{\text {nd }}$ International Simposium on Atmospheric Reentry Vehicles and Systems, Arcachon, France (2001). 


\section{http://dx.doi.org/10.21611/qirt.2006.b}

[14] F. De Filippis, S. Caristia, A. Del Vecchio, and C. Purpura, Scirocco final tests measured data: comparison between theory and experiments, Proceedings of the Fourth European Symposium on Aerothermodynamics for Space Vehicles, Capua, Italy (2001).

[15] F. De Filippis, S. Caristia, A. Del Vecchio, and C. Purpura., The Scirocco PWT Facility Calibration Activities, Proceedings of the $3^{\text {th }}$ International Symposium on Atmospheric re-entry vehicles and systems, Arcachon, France (2003).

[16] A. Del Vecchio, Hyflex test Campaign Report, SCIROCCO PROJECT, CIRA (2003).

[17] Y. Morimo, T. Yoshinaka, Reentry heating evalutation of thermal structure by use of the large arc heated wind tnnel facility (SCIROCCO), $8^{\text {th }}$ japan International SAMPLE Symposium , National Space Devolupementt Agency of Japan, NASDA (2003).

[18] G. Rufolo, D. Tescione and S. Borrelli, A Multidisciplinary Approch for the Analysis of Heat Shielded Space Structures, Italian Aerospace Research Center, CIRA (2003).

[19] F. Scortecci, F. Paganucci, L. d'Agostino and M. Andrenucci, A New Hypersonic High Enthalpy Wind Tunnel, 33rd Joint Propulsion Conference, AIAA 97-3017, Seattle (1997).

[20] F. Scortecci , F. Paganucci and L. Biagioni, Development of a Pulsed ArcHeater for a Hypersonic High Enthalpy Wind Tunnel, 33rd Joint Propulsion Conference, AIAA 97-3016, Seattle (1997). 